\title{
Microtaper Fiber Excitation Effects in Bottle Microresonators
}

\author{
M. Narizee Mohd Nasir, Ming Ding, G. Senthil Murugan and Michalis N. Zervas \\ Optoelectronics Research Centre, University of Southampton, Southampton, SO17 1BJ, U.K.
}

\begin{abstract}
We have carried out a systematic study of the effect of microtaper diameter on the spectral characteristics of bottle microresonators. By increasing the microtaper-diameter $\left(D_{t}\right)$ from $2 \mu \mathrm{m}$ to $10 \mu \mathrm{m}$ results in progressively cleaner spectra. The transmission depth at resonance varies from $\sim 15 \mathrm{~dB}\left(@ \mathrm{D}_{\mathrm{t}}=2 \mu \mathrm{m}\right)$ to $>3 \mathrm{~dB}\left(@ \mathrm{D}_{\mathrm{t}}=10 \mu \mathrm{m}\right)$. The loaded $\mathrm{Q}$ factors were measured to be $>10^{+6}$ in all cases. However, with microtaper $\mathrm{D}_{\mathrm{t}}=10 \mu \mathrm{m}$ clearly-resolved single resonance peaks could be observed and free-spectral ranges could be easily identified. At some transmission resonances, we have observed $\mathrm{LP}_{01} \rightarrow \mathrm{LP}_{11}$ mode transformation at the excitation microtaper waist, for the first time, as the resonance is scanned.
\end{abstract}

Keywords: Optical resonators, microcavity devices, optical sensing and sensors.

\section{INTRODUCTION}

Optical resonators are known to play a very crucial role in high performance laser devices and other photonic applications. Special designs are used to improve efficiency and output power performance ${ }^{1,2}$. An important limitation regarding traditional resonators is related to their weight, size, alignment requirements and environmental stability. Some of these problems are addressed by resorting to dielectric structures with circular symmetry and supporting whispering gallery modes (WGMs), without the need of additional reflecting arrangements. Optical microresonators which sustain WGMs are able to reach exceptionally high quality (Q) factors due to minimal leakage and scattering losses and low material absorption ${ }^{3}$. By tailoring the resonator size, shape and material composition, optical microresonators has shown great potential in being the basis of next generation compact size, low power and high speed photonics circuits. It has been widely demonstrated that optical microresonators are not only able to contribute towards fundamental research, but also in broad range of device applications which includes small-scale sensors, microlasers and filters, as a cavity quantum electrodynamics (CQED) and optical delay lines ${ }^{3,4}$. To address different applications, it is often critical to realize microresonators with compact size (small modal volume, V), high Q-factor and large free spectral range (FSR). Over the years, a very wide range of microresonator shapes has been explored with rotationally symmetric structures being widely used. Common type of the utilized shape includes cylinders, spheres, toroids and disks. Although cylindrical resonators had been shown to possess small Q-factor values, they can still be useful in some applications ${ }^{5}$. Ultra-high-Q-factor $\left(\sim 10^{10}\right)$ WGM resonator has been previously demonstrated through the utilization of silica microspheres ${ }^{6}$. However, the on-chip integration of this microresonator with other optical components is quite challenging ${ }^{7}$. It has been proposed that the challenge of microresonators on-chip integration could be overcome by the utilization of microring/disc resonators ${ }^{8}$. Nevertheless, Q-factors of such resonators are limited by the surface roughness introduced through its fabrication process.

Recently, a new type of optical microresonator - known as bottle microresonators (BMRs), has been given much attention due to its distinguishable features compared to other types of optical resonators. In contrast to microresonators mentioned above which rely predominantly on quasi-2D WGM optical confinement, BMRs show a truly 3D WGM confinement within its oblate structure ${ }^{9}$. BMRs also demonstrated a non-degenerate WGMs with an enhanced field strength corresponding to its modal turning points. Another interesting feature of BMRs is a rich variety of modes can be efficiently excited depending on the excitation arrangement ${ }^{10}$. We have previously demonstrated a simple and versatile way of fabricating BMRs through "soften-and-compress" technique ${ }^{10}$. As a result of strong asphericity of the BMR, dense and complex resonance spectral feature were generated due to degenerate supported modes with strongly overlapping FSRs and very large mode density ${ }^{11,12}$. Although the dense spectral features could be greatly beneficial for CQED studies, it would be a major drawback should the BMR is to be utilized for sensing purposes. Thus it is essential to have a "cleaner" spectrum with easily distinguishable and traceable features over a broad wavelength range. Previously, reduction to the complex and dense spectral features of BMRs has been proposed by attenuating a subset of

Laser Resonators, Microresonators, and Beam Control XV, edited by Alexis V. Kudryashov,

Alan H. Paxton, Vladimir S. Ilchenko, Lutz Aschke, Kunihiko Washio, Proc. of SPIE

Vol. 8600, 860020 - @ 2013 SPIE · CCC code: 0277-786X/13/\$18 - doi: 10.1117/12.2004282 
the BMRs' excited modes. This includes placement of micro-droplets ${ }^{13}$ and inscribing microgroove scars ${ }^{14}$ on BMRs. However, it is very hard to accurately position micro-droplets on BMRs in order to attenuate certain excited modes while ensuring the desired ones survive. Also, while microgroove scaring on BMRs could be very precise, it is too costly to be conventionally applied.

In this paper, we present a less constrained and cost-effective approach in generating cleaner and simpler spectra of BMRs through the manipulation of its excitation source. We study the effect of the diameter of the evanescently-coupled microtaper $\left(D_{t}\right)$ on the spectral characteristics of BMRs. Progressively cleaner and simpler spectra could be generated by BMRs when $D_{t}$ was increased from $2 \mu \mathrm{m}$ to $10 \mu \mathrm{m}$. The loaded Q-factors were also measured to be $>10^{+6}$ in all caseswhich is more than sufficient for sensing purposes. We also studied the modal transformation taking place at the microtaper waist as a result of coupling to BMR. It is observed that conversion of the $\mathrm{LP}_{01}$ microtaper mode into $\mathrm{LP}_{11}$ takes place in some of the strong transmission resonances in the case of 2 um diameters. The modal transformation was not as frequent in the case of 8um microtaper excitation, despite the much larger modality.

\section{EXPERIMENTAL CHARACTERIZATION}

The BMR utilized in this experiment was fabricated using the same "soften-and-compress" technique previously demonstrared ${ }^{10}$ with neck-to-neck distance $L_{b}$ of $360 \mu \mathrm{m}$, bottle diameter $D_{b}$ of $180 \mu \mathrm{m}$ and stem diameter $D_{s}$ of $125 \mu \mathrm{m}$. In this study, a standard telecom optical fiber (Corning SMF28) was used to fabricate the BMR. Figure 1 shows the BMR under study with dimensions summarized in the caption.

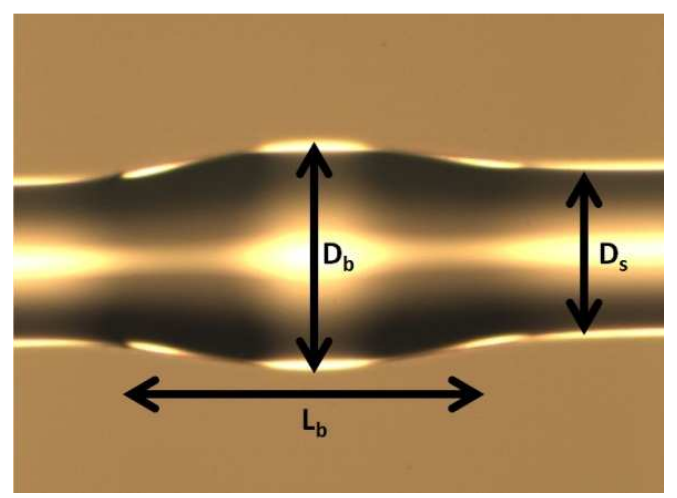

Figure 1. BMR under study with neck-to-neck diameter $L_{b}=360 \mu \mathrm{m}$, bottle diameter $D_{b}=180 \mu \mathrm{m}$ and stem diameter of $D_{s}=125 \mu \mathrm{m}$.

An important part of BMR study for sensing applications is the coupling of light into and out of the cavity. One of the best methods available for exciting the modes of the resonator cavity is through the utilization of microtaper fibers. With the right microtaper waist diameter, it is possible to couple $>90 \%$ of the input light into the cavity of a high-Q fused silica resonator ${ }^{15}$. For the case of microspheres, it has been reported that the optimum microtaper waist diameter is $\leq 2 \mu \mathrm{m}^{16}$ with coupling strength of $<1 \mathrm{~dB}$ observed when the microtaper waist diameter was increased to $>3 \mu \mathrm{m}$.

For this study, a total of five microtapers with waist diameter $D_{t}$ of $2,4,6,8$ and $10 \mu$ m were utilized to couple light into and out of the BMR. In each characterization process, the microtaper was positioned on top of the BMR with the utilization of micro-positioning stages. Although this overloads the cavity and would reduce the Q-factor, it results in a robust and stable arrangement. In order to observe the utilized BMR WGMs resonances, a tunable laser source TLS (Agilent $81600 \mathrm{~B}$ ) with linewidth of $\sim 100 \mathrm{kHz}$ was used to sweep a broad wavelength range. The output of the microtaper was monitored by an InGaAs detector. Images of the BMR being excited with the laser throughout different microtaper position were captured with an infra-red camera in order to observe light scattering caused by the BMR. Figure 2 shows the excitation at various microtaper positions along the BMR with $D_{t}$ of $10 \mu \mathrm{m}$. It can be clearly observed that, in contrast to most of the other demonstrated symmetrical optical resonators, BMR would give different modal 
intensity distributions at different excitation position. It has been theoretically predicted that excitation on BMR modes with characteristic intensity maxima on both sides of the corresponding turning point is observable as light is confined along the two turning points (along the $z$-axis) by angular momentum barrier ${ }^{9}, 17$ - as shown in Figure 2(e) and (g) where the microtaper was positioned $150 \mu \mathrm{m}$ away from the center. Positioning the microtaper beyond the bottle-neck (furthest turning point) caused no axial confinement of the excited modes, as in in Figure 2(f). As previously observed and discussed ${ }^{10}$, the BMR utilized in this experiment would also allow selective excitation of different modal families with different coupling position of the microtaper.

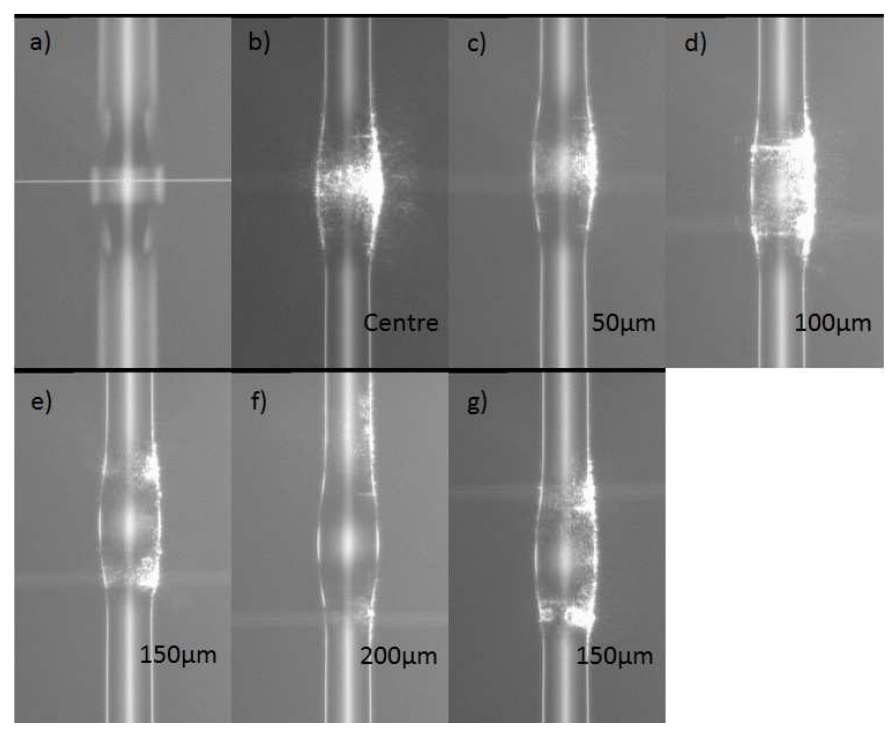

Figure 2. BMR images with microtaper of $\mathrm{D}_{t}=10 \mu \mathrm{m}$ positioned at (a) center without any input lasing light and (b) - (f) with input lasing light at center and $50 \mu \mathrm{m}, 100 \mu \mathrm{m}, 150 \mu \mathrm{m}$ and $200 \mu \mathrm{m}$ off-center respectively.

\section{EXPERIMENTAL RESULTS}

Figures 3(a) - (e) show transmission spectra of the utilized BMR when coupled and excited at the center with microtaper waist diameter $D_{t}$ of $2,4,6,8$ and $10 \mu \mathrm{m}$. With $D_{t}=2 \mu \mathrm{m}$, the transmission depth gained is $\sim 15 \mathrm{~dB}$. It decreases to $\sim 13 \mathrm{~dB}$ when $\mathrm{D}_{t}=4 \mu \mathrm{m}$ and $6 \mu \mathrm{m}$. This is expected since microtaper with larger $\mathrm{D}_{t}$ allows more light to propagate in the core than the one with smaller Dt. Hence there is less light available that is propagating in the cladding-air region as evanescent field to be coupled into the BMR. Transmission depth to the BMR decreases further to $\sim 10 \mathrm{~dB}$ when $\mathrm{D}_{\mathrm{t}}$ $=8 \mu \mathrm{m}$. With $\mathrm{D}_{\mathrm{t}}=10 \mu \mathrm{m}$, coupling efficiency into the BMR dropped to $\sim 3 \mathrm{~dB}$. BMR WGMs are defined by $m$ (azimuthal), $\rho$ (radial) and $q$ (axial) mode numbers and characteristic wavelength $\lambda_{m \rho q}$. In contrast with near-perfect-microspheres, azimuthal mode degeneracy is broken in BMRs and the strong overlap of different mode families' result in very dense and complex transmission spectra ${ }^{10-12}$.

It could be clearly observed that progressively cleaner and simple spectra could be generated by the utilized BMR as the microtaper waist diameter $D_{t}$ increases from 2 to $10 \mu \mathrm{m}$. The observed spectral dependencies on microtaper diameter is believed to be due to the fact that smaller microtaper diameter show smaller effective indices and therefore phasematch higher-radial-order BMR WGMs. Owing to strong modal-family overlaps, the density of modes becomes progressively larger as the radial order increases- resulting in complex and dense transmission spectra. With larger excitation microtaper diameters $(>6 \mu \mathrm{m})$, cleaner spectra of the utilized BMR could be generated as phase matching to lower-radial-order BMR WGMs takes place. With microtaper $D_{t}=10 \mu \mathrm{m}$, clearly resolved single resonance peaks could be identified and hence much distinguishable FSRs observed. The generated single resonance peaks from the incorporated BMR and large microtaper $\mathrm{D}_{\mathrm{t}}(>6 \mu \mathrm{m})$ would be greatly beneficial for sensing purposes as indication markers for any wavelength shifts. In addition, larger microtaper diameters result in a much more robust devices 
showing negligible deterioration with environmental effects, such as humidity. Microtapers with $2 \mu \mathrm{m}$, on the other hand, are known to deteriorate quickly resulting in large surface scattering and transmission drop. Comparing Figures 2 (a) and (e) show that utilization of $10 \mu \mathrm{m}$ microtaper would results in an increase of the throughput power by $>2 \mathrm{~dB}$.

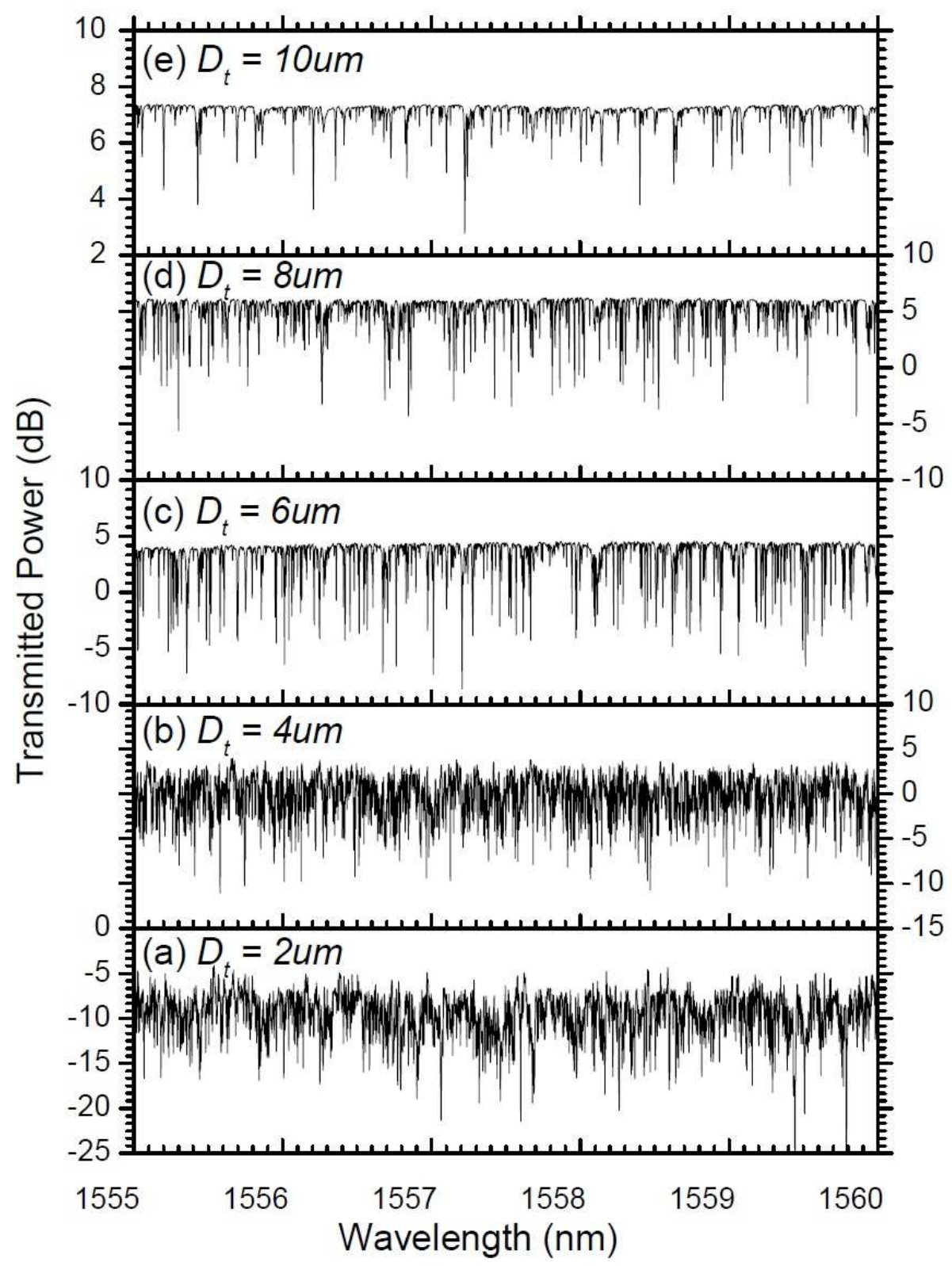

Figure 3. Resonance spectra of the excited BMR when the microtapers are positioned at the center with $D_{t}$ of (a) $2 \mu \mathrm{m}$, (b) $4 \mu \mathrm{m},(\mathrm{c}) 6 \mu \mathrm{m},(\mathrm{d}) 8 \mu \mathrm{m}$ and (e) $10 \mu \mathrm{m}$.

Figure 4 illustrates specific resonant feature of the BMR when excited at center position with microtaper $D_{t}$ of 2,4 , 6,8 and $10 \mu \mathrm{m}$. The wavelength span is varied in order to best demonstrate the observed Q-factor. Q-factor is defined by $\lambda / \Delta \lambda$ (where $\lambda$ is the wavelength at which a resonance occurs and $\Delta \lambda$ is the linewidth of the resonant wavelength). Lorentzian fit for each measured data were also performed in order to calculate the Q-factor of the dominant resonances. In all cases, the loaded Q-factor were measured to be $>10^{+6}$. Results from this study indicate that microtaper waist diameter does not play much influence on the Q-factor value of the utilized BMR. 

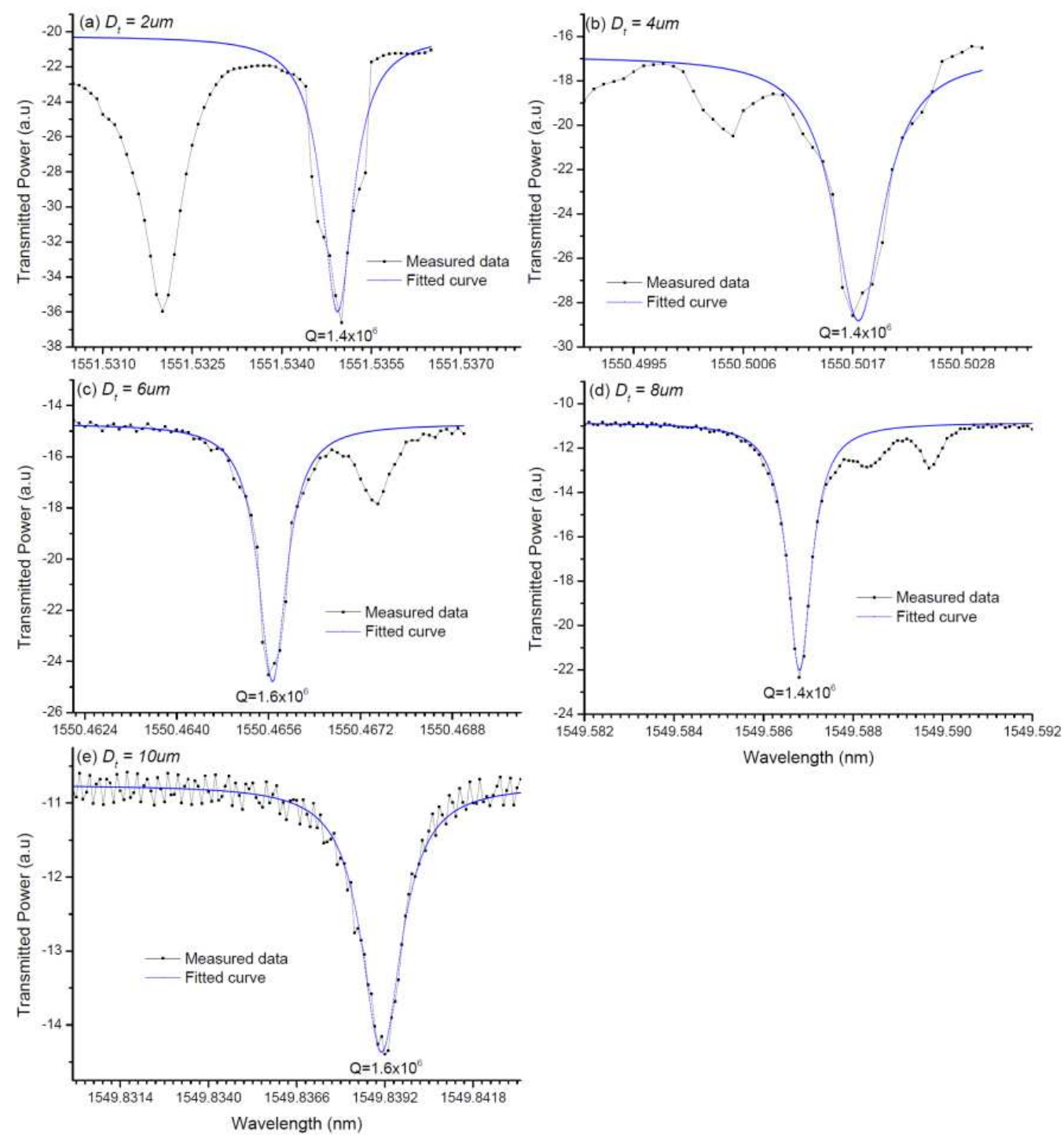

Figure 4. Measured data and Lorentzian fit of individual transmission resonances with $D_{t}$ of (a) $2 \mu \mathrm{m}$, (b) $4 \mu \mathrm{m}$, (c) $6 \mu \mathrm{m}$, (d) $8 \mu \mathrm{m}$ and (e) $10 \mu \mathrm{m}$.

\section{MODAL TRANSFORMATION OF BOTTLE MICRORESONATOR}

The microtapers used for the $\mathrm{BMR}$ excitation are fabricated to be adiabatic and therefore the launched $\mathrm{LP}_{01}$ fundamental mode is maintained throughout its length. However, along its waist, where the original core is diminished and light is guided by the waist itself, the microtaper supports a number of modes. In the presence of external perturbations, such as the contacting $\mathrm{BMR}$, higher order modes are likely to be excited. In such case, the input $\mathrm{LP}_{01}$ mode is expected to be (partially) transformed into higher order "waist modes". Previous studies have suggested that higher-order mode transformation will compromise the coupling 'ideality' of microtapers to microresonators and this effect is expected to become worse as $D_{t}$ increases ${ }^{18}$. Modal transformation from the excitation source to BMR was experimentally investigated through near field images of the output beam. The study was performed by utilizing microtapers of $D_{t}=2 \mu \mathrm{m}$ and $8 \mu \mathrm{m}$. The microtapers were cleaved at their respective minimum-waist regions and placed on top of the BMR at center position. Figure 5(a) shows light scattering of the BMR when terminated microtaper of $D_{t}$ $=8 \mu \mathrm{m}$ was connected to a HeNe laser source. 

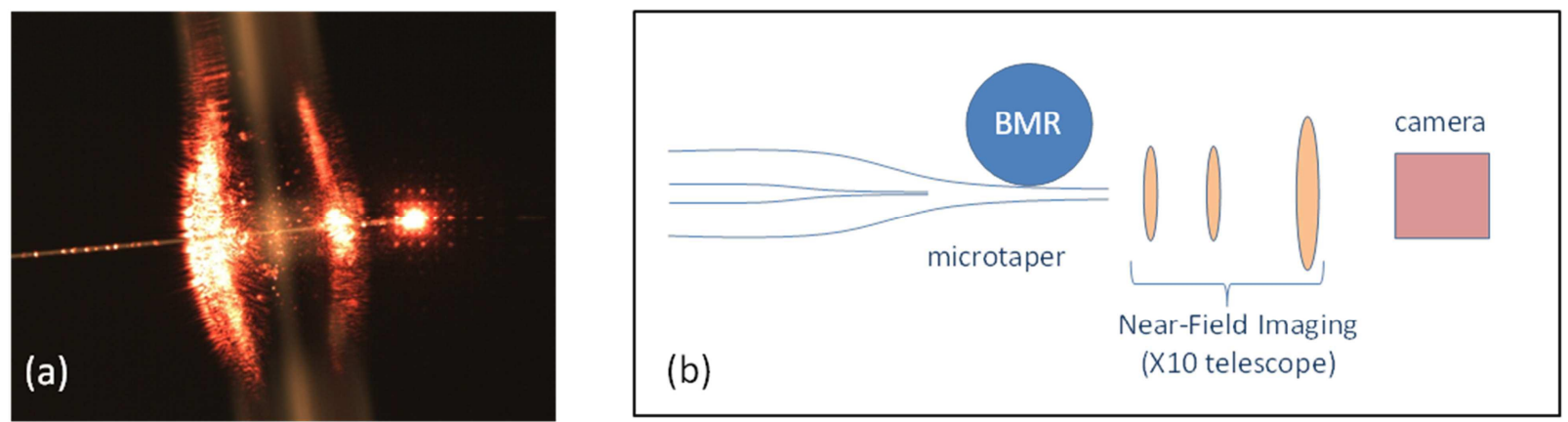

Figure 5. (a) Light scattering of BMR with terminated microtaper $\left(D_{t}=8 \mu \mathrm{m}\right)$ connected to a HeNe laser source. (b) Cleaved microtaper waist and $\mathrm{x} 10$ telescope schematic for modal transformation visualization.

Figure 5(b) shows a schematic of the X10 telescope used to visualize modal transformations at various transmission resonances. A collimator was placed at the end of the terminated microtaper in order to capture the output light. Before capturing the output beam, BMR WGMs resonance spectra were first measured by placing a lens-to-fiber connector in front of the collimator and into the InGaAs power meter. For the imaging process, an infra-red camera was utilized to capture the output beams. The output beams were magnified X10 with the incorporation of two bi-convex lenses in order to clearly observe any modal transformation.

(a)

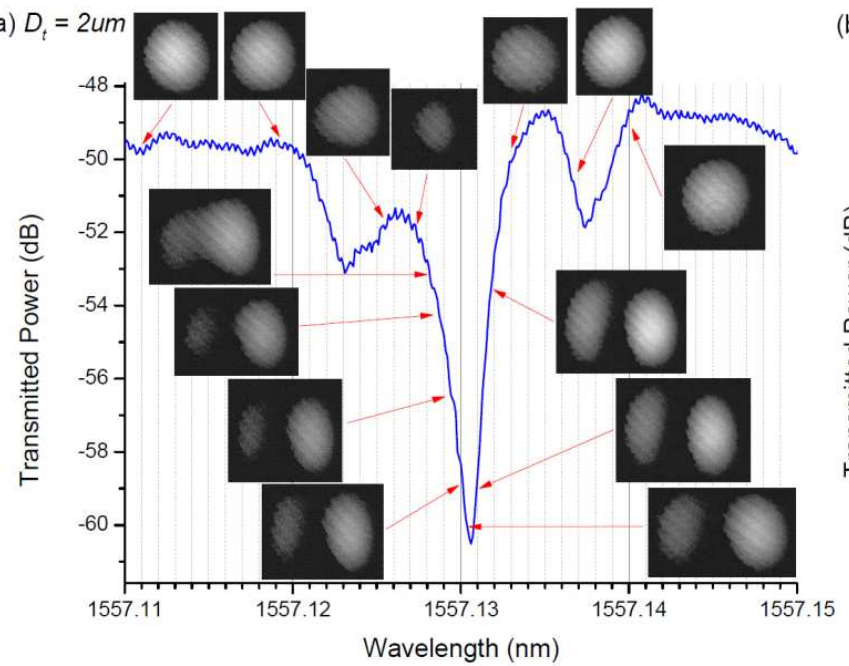

(b) $D_{t}=8 u m$

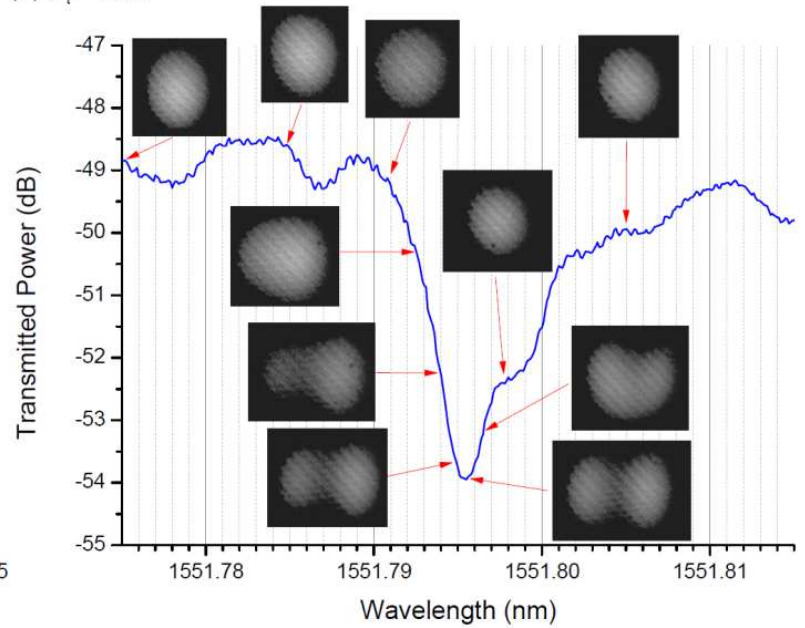

Figure 6. Near field images of BMR output beam through steps of finely tuned laser source at one specific resonance peak with $\mathrm{D}_{\mathrm{t}}$ of (a) $2 \mu \mathrm{m}$ and (b) $8 \mu \mathrm{m}$.

Figure 6 illustrates the observed BMR modal transformation at one specific resonance using a finely tuned wavelength laser source. Figure 6(a) illustrates the BMR modal transformation with microtaper $D_{t}=2 \mu \mathrm{m}$ at resonance wavelength of $1557.131 \mathrm{~nm}$. Off-resonance (between $1557.110-1557.120 \mathrm{~nm}$ ), only the fundamental $\mathrm{LP}_{01}$ mode were observed. Around $1557.123 \mathrm{~nm}$, there is a group of minor simultaneously excited WGMs. Near field image at this point shows the output beam became slightly distorted-due to the different modes beating. At around $1557.127 \mathrm{~nm}$, the output beam started to lose power due to efficient light coupling into the BMR. At this strong resonance point, the TLS power was increased in order to have more light out of the microtaper for adequate image processing. The beating of LP $\mathrm{P}_{11}$ mode started to become significant at $\sim 1557.128 \mathrm{~nm}$. Tuning the wavelength further causes the $\mathrm{LP}_{11}$ mode to become more and more dominant. Modal transformation from $\mathrm{LP}_{01}$ to $\mathrm{LP}_{11}$ was clearly observed at resonance peak, which is at 
$\sim 1557.131 \mathrm{~nm}$ point. Again, off resonance $(\sim 1557.133 \mathrm{~nm})$, only the $\mathrm{LP}_{01}$ fundamental mode observed. With an exception of a few, most of the remaining strong WGM resonances showed noticeable $\mathrm{LP}_{11}$ mode excitation at their peak resonance wavelength.

In contrast with the findings above, with the microtaper of $D_{t}=8 \mu \mathrm{m}$, only a few of the BMR WGM resonances checked show clear $\mathrm{LP}_{01}$ to $\mathrm{LP}_{11}$ modal transformation. Most of the strong resonances only show distortion on the output beam image- an indication of two modes beating. Some of the resonances checked did not even show any noticeable LP $_{11}$ mode excitation. Nevertheless, as shown in Figure $6(\mathrm{~b})$, the few resonances which did show clear modal transformation follow the same trend as with the previous microtaper. At off-resonance, only the fundamental LP $\mathrm{P}_{01}$ mode observed. The mode beating of $\mathrm{LP}_{01}$ and $\mathrm{LP}_{11}$ became more and more significant when the wavelength was tuned near to the peak resonance point. The mode then transformed to $\mathrm{LP}_{11}$ at peak resonance $(\sim 1551.795 \mathrm{~nm})$ and back again to $\mathrm{LP}_{01}$ at off-resonance. The resonance shown in Figure 6(b) does consist of another simultaneous WMG excitation at $\sim 1551.798 \mathrm{~nm}$. Hence the output beam profile at this point will also be influenced by the presence of this second excited WGM.

Previous studies suggested that coupling 'ideality' of microtapers to microresonators would decrease as $D_{t}$ increases ${ }^{18}$. Strong modal transformations have been observed for the smaller waist diameter microtaper. However, despite the fact that larger waist diameter microtapers support much larger number of modes, the observed modal transformation was significantly smaller. This implies that modal phase-matching, rather than modal number defines the degree of modal transformation in evanescently coupled microtapers.

\section{CONCLUSION}

We have studied the effect of microtaper waist diameters on the evanescent-filed excitation of BMRs. Demonstration of BMR spectra "clean-up" has been successfully demonstrated through the manipulation of the excitation microtaper waist diameter. The utilization of microtapers with relatively large waist diameter $(>6 \mu \mathrm{m})$ was observed to generate much simpler spectra due to phase matching of lower-radial-order BMR WGMs. This would be greatly beneficial for sensing purposes. Even with microtaper of $D_{t}=10 \mu \mathrm{m}$, coupling efficiency of more than $50 \%$ into the BMR has been shown possible. This is in contrast with the previously studied case of near-perfect-microspheres where microtapers with $D_{t} \leq 2 \mu \mathrm{m}$ are considered to be optimum. Microtaper waist diameters were also shown to have little influence on the loaded Q-factor values of the BMR. All of the measured Q-factor values with different $D_{t}$ size were observed to be $>10^{+6}$.

Modal transformations of BMRs were also experimentally investigated by the observation of near-field images of the output beam. Most of the strong WGM resonances checked with microtaper of $D_{t}=2 \mu m$ show clear transformation from fundamental $\mathrm{LP}_{01}$ mode to higher order $\mathrm{LP}_{11}$ mode. It should be further noted, that due to the following adiabatic up-taper, the $\mathrm{LP}_{11}$ mode will be radiated away and will not captured by the single-mode core of the interrogating fiber. Depending on the degree of BMR-induced modal transformation, this will result in apparently stronger resonance dips and enormous coupling strengths. However, this transformation is not so pronounced and frequent with microtaper of $D_{t}=8 \mu \mathrm{m}$ where only a few strong resonances investigated show clear modal transformation from lower to higher order. Hence there is no major concern of coupling losses from lower order modes to higher order modes on BMRs by utilizing microtaper with higher $D_{t}$.

\section{REFERENCES}

[1] D. Hall and Jackson P., "The Physics and Technology of Laser Resonators”. Taylor and Francis (1989).

[2] Hodgson N. and Weber H., "Laser Resonators and Beam Propagation", $2^{\text {nd }}$ Edition, Springer (2005).

[3] K. J. Vahala, "Optical Microcavities", Nature, 424, 839-846 (2003).

[4] V. S. Ilchenko and A. B. Matsko, "Optical Resonators with Whispering-Gallery Modes -Part II: Applications," IEEE Journal of Selected Topics in Quantum Electronics, 12, 15-32 (2006).

[5] T. A. Birks, J. C. Knight and T.E. Dimmick, "High-Resolution Measurement of the Fiber Diameter Variations Using Whispering Gallery Modes and No Optical Alignment", IEEE Photonics Technology Letters, 12, 182-183 (2000).

[6] M. L. Gorodetsky, A. A. Savchenkov, and V. S. Ilchenko, "Ultimate Q of optical microspheres resonators", Optics Letters, 21, 453-455 (1996). 
[7] G. S. Murugan, J. S. Wilkinson and M. N. Zervas, "Optical microdiscus resonator by flattening microspheres", Applied Physics Letters, 101, 071106-071106-4 (2012).

[8] B. E. Little, J. S. Foresi, G. Steinmeyer, E. R. Thoen, S. T. Chu, H. A. Haus, E. P. Ippen, L. C. Kimerling, and W. Greene, "Ultra-compact Si-SiO2 microring resonator optical channel dropping filters", IEEE Photonics Technology Letters, 10, 549-551 (1998).

[9] M. Sumetsky, "Whispering-gallery-bottle microcavities: the three dimensional etalon", Optics Letters, 29, 8-10 (2004).

[10] G. S. Murugan, J. S. Wilkinson, and M. N. Zervas, "Selective excitation of whispering gallery modes in a novel bottle microresonator", Optics Express, 17, 11916-11925 (2009).

[11] M. L. Gorodetsky, and A. E. Fomin, "Geometrical theory of whispering-gallery modes", IEEE Journal of Selected Topics in Quantum Electronics, 12, 33-39 (2006).

[12]G. Chen, Md. M. Mazumder, R. K. Chang, J. C. Swindalt, and W. P. Ackert, "Laser diagnostics for droplet characterization: application of morphology-dependent resonances," Progress in Energy and Combustion Science, 22, 163-188 (1996).

[13]G. S. Murugan, M. N. Petrovich, Y. Jung, J. S. Wilkinson, and M. N. Zervas, "Hollow-bottle optical microresonators", Optics Express, 19, 20773-20784 (2011).

[14] M. Ding, G. S. Murugan, G. Brambilla, and M. N. Zervas, "Whispering gallery mode selection in optical bottle microresonators", Applied Physics Letters, 100, 081108 (2012).

[15] J. C. Knight, G. Cheung, F. Jacques, and T. A. Birks, "Phased-matched excitation of whispering-gallery-mode resonance by a fiber taper", Optics Letters, 22, 1129-1131 (1997).

[16] O. Svitelskiy, Y. Li, A. Darafsheh, M. Sumetsky, D. Carnegie, E. Rafailov, and V. N. Astratov, "Fiber coupling to $\mathrm{BaTiO}_{3}$ glass microspheres in an aqueous environment", Optics Letters, 36, 2862-2864 (2011).

[17] Y. Louyer, D. Meschede, and A. Rauschenbeutel, "Tunable whispering-galery-mode resonators for cavity quantum electrodynamics", Physical Review, 72, 031801(R), (2005).

[18] S. M. Spillane, T. J. Kippenberg, O. J. Painter, and K. J. Vahala, "Ideality in a Fiber-Taper-Coupled Microresonator System for Application to Cavity Quantum Electrodynamics”, Physical Review Letters, 91, 043902 (2003). 